\section{False positive phencyclidine result on urine drug testing: a little known cause}

Phencyclidine (PCP) is a hallucinogenic drug, often referred to as 'angel dust'. Its short-term effects are seen for approximately $1 \mathrm{~h}$ after ingestion and may include hallucinations, disinhibition, euphoria and agitation. Long-term use can lead to symptoms resembling psychotic disorders such as schizophrenia. Its detection time in urine is approximately 8 days. ${ }^{1}$ We would like to highlight two cases of false positive results for PCP on urine drug screening at a community mental health rehabilitation centre.

Patient $A$ was a 25 -year-old male with paranoid schizophrenia, admitted to an acute psychiatric ward under Section 2 of the Mental Health Act 1983 because of deterioration in mental state following medication nonadherence and a history of illicit drug use. He was transferred to the rehabilitation centre under Section 3 of the Act 5 months later, exhibiting mainly negative symptoms of schizophrenia. He was receiving treatment with venlafaxine $150 \mathrm{mg}$ twice daily, lithium carbonate $800 \mathrm{mg}$ once daily and clozapine $400 \mathrm{mg}$ in the evening; he also had lactulose $10 \mathrm{ml}$ twice daily. A urine drug screen was performed after staff found cannabis in his room. The result was positive for both PCP and THC (marijuana), although the patient denied taking any PCP. The test was repeated and results were positive for PCP only.

Patient B was a 38-year-old male with paranoid schizophrenia admitted under Section 2 of the Mental Health Act after being arrested for wielding knives in public. He was transferred to the rehabilitation centre under Section 3 of the Act 8 months later with ongoing psychotic symptoms including 'electric shock sensations' which he attributed to possible chemical warfare. He was receiving treatment with risperdal consta $50 \mathrm{mg} \mathrm{IM}$ twice weekly, venlafaxine $75 \mathrm{mg}$ twice daily, clonazepam $0.5 \mathrm{mg}$ twice daily and procyclidine $5 \mathrm{mg}$ twice daily. A urine drug screen was performed since he had become increasingly guarded and irritable, despite good adherence to medication. The result was positive for PCP and benzodiazepines. The benzodiazepines could be explained by clonazepam but the patient again denied taking any PCP. The same results were obtained when the test was repeated.

Given that both patients denied taking PCP our suspicion was aroused. None of the other patients on the unit who had urine drug screens tested positive for PCP. Venlafaxine was the only medication taken by both patient $A$ and $B$. A review of the literature revealed several case reports of false positive urine immunoassay results for PCP in patients taking venlafaxine of various doses. In one case series, three patients in an emergency department in Danbury Hospital, Connecticut, USA, were found to have false positive urine assay results for PCP due to venlafaxine. ${ }^{2}$ Another case reported a false positive result for $\mathrm{PCP}$ in a patient with an intellectual disability who received $75 \mathrm{mg} / \mathrm{d}$ of venlafaxine extended-release $(X R)^{3}$ and another that resulted from venlafaxine overdose. ${ }^{4}$

This effect is thought to be due to cross-reactivity between venlafaxine and the active metabolite O-desmethylvenlafaxine with the PCP assay reagent, although they are not structurally related. ${ }^{2}$ The US Food and Drug Administration warns that false positive test results may be expected for several days following discontinuation of venlafaxine. ${ }^{5}$
Confirmatory tests, such as gas chromatography/mass spectrometry can be used to distinguish between the two.

Based on this information, the urine assay results showing $\mathrm{PCP}$ for patients $\mathrm{A}$ and $\mathrm{B}$ were determined to be false positives due to cross-reactivity with venlafaxine. Patient A's leave was reinstated as it had been cancelled until drug testing was negative. For patient $B$, we were able to exclude illicit drug use as a cause for his altered mental state. Increased awareness of the cross-reactivity between PCP and venlafaxine is important for all healthcare professionals to avoid inappropriate suspicion of illicit drug use.

1 Moeller KE, Lee KC, Kissack JC. Urine drug screening: practical guide for clinicians. Mayo Clin Proc 2008; 83: 66-76

2 Sena SF, Kazimi S, Wu AH. False-positive phencyclidine immunoassay results caused by venlafaxine and $\mathrm{O}$-desmethylvenlafaxine. Clin Chem 2002; 48: 676-7.

3 Brahm NC, Brown RC. Venlafaxine usage resulted in a false positive immunoassay for phencyclidine. J Coll Psychiatr Neurol Pharm (http:// cpnp.org/_docs/resource/jcpnp/venlafaxine.pdf).

4 Bond GR, Steele PE, Uges DR. Massive venlafaxine overdose resulted in a false positive Abott AXSYM urine immunoassay for phencyclidine. $J$ Toxicol Clin Toxicol 2003; 41: 999-1002.

5 U.S. Food and Drug Administration. Effexor (venlafaxine $\mathrm{HCl}$ ) tablets and Effexor XR (venlafaxine $\mathrm{HCl}$ ) extended-release capsule. FDA (http://www.fda.gov/Safety/MedWatch/Safetylnformation/ ucm201064.htm).

Gabriella L. Landy is a core trainee (CT3) psychiatry, email: gabriella.landy@candi.nhs.uk, and Mukesh Kripalani is a consultant psychiatrist, both at Camden and Islington NHS Foundation Trust, London, UK.

\section{doi: $10.1192 / \mathrm{pb} \cdot 39.1 .50$}

\section{No beds for young people - also in Scotland}

I read Myers et al's correspondence ${ }^{1}$ with great interest. I am a consultant child and adolescent psychiatrist working in the forensic child and adolescent mental health services and I am simply dumbfounded by the difficulties that frequently present when trying to coordinate in-patient admissions for young people in Scotland for those who have mental health problems and concurrent risk to others.

Like the authors of the letter, I see the deterioration and the stigma that young people face when admissions are being coordinated. At present, there are no secure mental health beds in Scotland who accept under-18-year-olds. Our only option is to beg for intensive psychiatric care unit beds from colleagues in adult services. I also echo concerns that there is no joined-up bed management system within the service I work for, which means that should I wish to admit a young person, it is up to me to call each unit individually.

Often my only option is to send young people to England, where there are private-sector adolescent medium secure beds. This comes with significant cost, both financial and emotional. I have seen how hard it is for families to agree to send their loved ones so far away, knowing they will struggle to visit or sometimes even telephone. In addition, if a young person is on remand or pre-trial, they cannot be sent across the border.

I thank the authors for making me realise that I am not isolated in this demoralising and stigmatising situation. But this is a bittersweet pill as it only serves to highlight that services need to be made more available for young people across the country. 
1 Myers G, Coyle D, Kowalski C, Srinivasan R. How can a young person wait over 90 hours in an emergency department for a bed? Psychiatr Bull 2014; 38: 250 .

Laxmi Kathuria is a consultant psychiatrist working at Yorkhill Hospital, Glasgow, UK, email: laxmi.kathuria@nnhs.net.

doi: $10.1192 / p b .39 .1 .50 a$

\section{It is more than just beds}

We read with interest the correspondence by Myers et al ${ }^{1}$ and echo their concern. In our region, child and adolescent psychiatrists are increasingly dealing with similar situations and are concerned for young people and their experience of services out of hours. We agree that there is no current system to find out bed availability and no external support to make this process efficient.

Fortunately, in our region we have an out-of-hours process whereby referrals can be made and we have agreement for two tier 4 providers to accept emergency admissions. Since this process was initiated, the referrals for out-of-hours beds have steadily increased and in the past 6 months 30 referrals were made, two-thirds of which were for people aged 17+. However, despite this process, only five young people were able to access these emergency beds in that period. The majority of young people had to wait until NHS England was available to manage the referral the next working day. Hence, there have also been calls in our region for daily bed state availability and for NHS England to be accessible out of hours.

Ensuring the best use of a scarce resource and the prioritisation of available beds requires high-quality and skilled clinical assessment. We also provide a gateway service/access assessment during working hours. This has averted the need for in-patient admission for a third of patients referred. It has been valued by referrers and ensures that the right patient accesses the right type of service. However, this service is not available out of hours.

We agree that increased bed provision is not the only solution. The divide in commissioning arrangements for tier 3 and tier 4 services means the development of alternatives to in-patient admission; outreach and crisis services and daypatient services have been patchy, too. In Birmingham we have developed a child and adolescent mental health home treatment service that has demonstrated a reduction in need for admission and cut length of stay by $50 \%$. Birmingham has also set up daytime and out-of-hours community emergency response and assessment teams that respond to emergency referrals from all the local general hospitals.

The report published by the Health Select Committee on 5 November 2014 highlights this major problem with access to in-patient services, as well as problems with commissioning and the lack of services which bridge the gap between inpatient and out-patient services. ${ }^{2}$ It takes a whole-systems view and recognises that the problem is about more than just beds.

1 Myers G, Coyle D, Kowalski C, Srinivasan R. How can a young person wait over 90 hours in an emergency department for a bed? Psychiatr Bull 2014; 38: 250 .
2 House of Commons Health Committee. Children's and Adolescents' Mental Health and CAMHS. Third Report of Session 2014-15. TSO (The Stationery Office) (http://www.publications.parliament.uk/pa/ cm201415/cmselect/cmhealth/342/342.pdf).

Nicole K. Fung is a consultant child and adolescent psychiatrist at Heathlands Unit, email: nicole.fung@bch.nhs.uk, and Linda Cullen is clinical service director at Tier 4 CAMHS, Birmingham Children's Hospital NHS Foundation Trust, Parkview Clinic, Birmingham, UK.

doi: $10.1192 / p b .39 .1 .51$

\section{Self-diagnosing bipolar disorder: questions for clinicians}

It is not uncommon in psychiatry to receive referrals for patients who believe they have bipolar affective disorder. This has been explained partly by a trend of celebrities openly talking about having bipolar disorder along with an explosion of information about bipolar illness on the internet. ${ }^{1}$

We analysed the records of 46 patients who over a 3-year period requested referral to a community mental health team seeking a diagnosis of bipolar illness. The patients were predominantly young women (mean age 32 years, female:male ratio $31: 15)$. Clinically, they presented with problems of anxiety and low mood with a history of mood swings (90\%), racing thoughts (70\%), impulsivity (100\%) and overactivity (60\%). All patients had visited a website offering self-assessment for bipolar illness and reported scores being highly suggestive of a bipolar illness - this had influenced their decision to seek referral. Around $25 \%$ of patients reported seeing a TV programme featuring a celebrity talking about their bipolar illness. Five patients, of their own accord, had joined their local Bipolar UK support group before the assessment.

None of the patients were given the diagnosis of bipolar illness at initial assessment. All were given formulations about their problems in terms of mood swings, coping and lifestyle issues. The ICD-10 diagnostic categories were mixed anxiety depression/adjustment disorder/dysthymia (20 patients); emotionally unstable personality disorder (10); alcoholism/ alcohol misuse (5); no psychiatric diagnosis (11). About a third of patients, after having their history taken, readily agreed at the end of the first meeting that they were not suffering from a bipolar illness. Five patients asked for a second opinion; all were experiencing relationship problems.

Our experience highlights the issues that may be encountered while assessing patients who actively seek diagnosis of a bipolar illness. There is merit in taking the patient into confidence about the confusion surrounding diagnosing bipolar illness and the risks associated with medical treatment. Also, while trying to arrive at a diagnosis, it may be best to look for classical or severe bipolar illness and if the evidence is not strongly suggestive then the diagnosis should be avoided or deferred until conclusive evidence is obtained.

1 Chan D, Sireling L. 'I want to be bipolar' ... a new phenomenon. Psychiatrist 2010; 34:103-5.

Avneet Sharma is a consultant psychiatrist with South Gloucestershire Recovery Team, Blackberry Hill Hospital, Bristol, UK, email: avneet. sharma@nhs.net, and Jitender Kumar is a speciality trainee (ST5) in forensic psychiatry at Fulbourne Hospital, Cambridge.

doi: 10.1192/pb.39.1.51a 